\title{
Holography for Heavy lons Collisions at LHC and NICA
}

\author{
Irina Aref'eva ${ }^{1, a}$ \\ ${ }^{1}$ Steklov Mathematical Institute, Russian Academy of Science
}

\begin{abstract}
This is a contribution for the Proceedings of 5th International Conference on New Frontiers in Physics (ICNFP 2016), held at Crete, 6-14 July 2016. Our goal is to obtain phenomenologically reliable insights for the physics of the quark-gluon plasma (QGP) from the holography. I briefly review how in the holographical setup one can describe the QGP formation in heavy ion collisions and how to get quantitatively the main characteristics of the QGP formation - the total multiplicity and the thermalization time. To fit the experimental form of dependence of total multiplicity on energy, obtained at LHC, we have to deal with a special anisotropic holographic model, related with the Lifshitz-type background.

Our conjecture is that this Lifshitz-type background with non-zero chemical potential can be used to describe future data expected from NICA. In particular, we present the results of calculations the holographic confinement/deconfinement phase transition in the $(\mu, T)$ (chemical potential, temperature) plane in this anizotropic background and show the dependence of the transition line on the orientation of the quark pair. This dependence leads to a non-sharp character of physical confinement/deconfinement phase in the $(\mu, T)$-plane. We use the bottom-up soft wall approach incorporating quark confinement deforming factor and vector field providing the non-zero chemical potential. In this model we also estimate the holographic photon production.
\end{abstract}

\section{Introduction}

Quark Gluon Plasma (QGP) produced in heavy ion collisions (HIC) at RHIC and LHC is a new form of matter formed from quarks and gluons at hight temperature [1, 2]. It is a strong coupling fluid [3], or an "operator boiling fluid" [4]. This makes perturbative methods inapplicable to study properties of QGP and especially it formation. The lattice QCD, the powerful tool of non-perturbative study, is formulated in the Euclidean spacetime and cannot be applied to QGP formation, since this is time depending phenomena (cf.[5]). This gives a strong motivation to study the process of QGP formation in HIC through the gauge/string duality. The gauge/string duality (or AdS/CFT correspondence) is directly applicable only for a very special 4-dimensional theory, namely, $\mathcal{N}=4$ SUSY Yang Mills theory [6]. This theory is conformal invariant on the quantum level and the conformal invariance is the main request for the AdS/CFT application. Of course, the real QCD is not conformal invariant, however, as we know from lattice calculations, the high-temperature QCD is. More precise, the deviation from conformality decreases as the temperature increases, see Fig.1.A. In Fig.1.B the QCD phase diagram is presented. The phase diagram of QCD is not well known either experimentally or

ae-mail: arefeva@mi.ras.ru 
theoretically. A commonly conjectured form of the phase diagram, temperature $\mathrm{T}$ vs quark chemical potential $\mu$, is shown in Fig.1.B. The chemical potential $\mu$ is a measure of the imbalance between quarks and antiquarks in the system. The phase transition is not sharp and it is supposed to be the 1-st order.

Ordinary nuclear matter in this diagram is at $\mu=310 \mathrm{MeV}$ and $\mathrm{T}$ close to zero. If we increase the quark density, i.e. increase $\mu$, keeping the temperature low, we go into a phase of more and more compressed nuclear matter neutron stars. Above the (blue on the on-line version of the paper) smeared line there is a transition to the quark-gluon plasma. At ultra-high densities one expects to find the phase of color-superconducting quark matter. In ultra-relativistic heavy ion collisions one studies this matter in the regime of extreme energy density. In Fig.1.B. the typical values of $\mu$ and $T$ in heavy-ion collisions (RHIC and LHC) are shown by a filled (cyan) region near the T-axis. The regions expected to be available at NICA (Nuclotron-based Ion Collider fAcility) [7] and FAIR (Facility for Antiproton and Ion Research) are indicated by arrows.

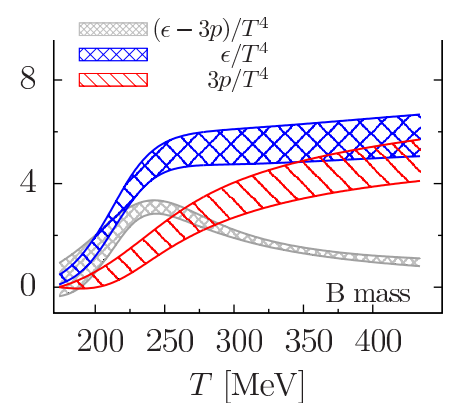

A

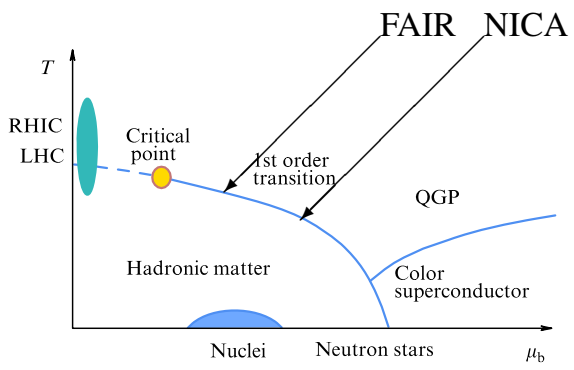

$\mathrm{B}$

Figure 1. A. The trace anomaly, energy density and pressure for two flavors of twisted mass Wilson fermions at $m_{\pi}=360 \mathrm{MeV}$. The plot from [8]. B.The QCD phase diagram

The exact dual description of the real QCD is unknown, but holographic QCD models that fit perturbative (two loops $\beta$-function) and lattice QCD results (in particular, the quark confinement potential) have been proposed $[9,10]$. Using these models several static properties of QGP have been reproduced [11].

The description of the QGP formation in HIC is a difficult subject, since it supposes to study a complicated real time phenomena - thermalization. We also do not know much from experiments about the details of the QGP formation in HIC, one can just estimates the time of QGP formation as well as the total multiplicity (there are arguments that the main part of particles is produced during the QGP formation) $[12,13]$. The QGP formation has been the subject of the active studies within holographic approach in last years (see [14-16] and refs therein). Initially this problem has been considered in AdS background [17-23] and the total multiplicity within this approach has been estimated as

$$
\mathcal{M}_{\text {AdS }} \sim s^{0.33}
$$

For the improved holographic background the estimation was [24]

$$
\mathcal{M}_{I H Q C D} \sim s^{0.22}(1+\log \text { corrections }),
$$


The experimental multiplicity dependence on energy $[12,13]$ is

$$
\mathcal{M}_{L H C} \sim s^{0.155}
$$

and it has been shown in [25] that the dependence (3) requires an unstable background.

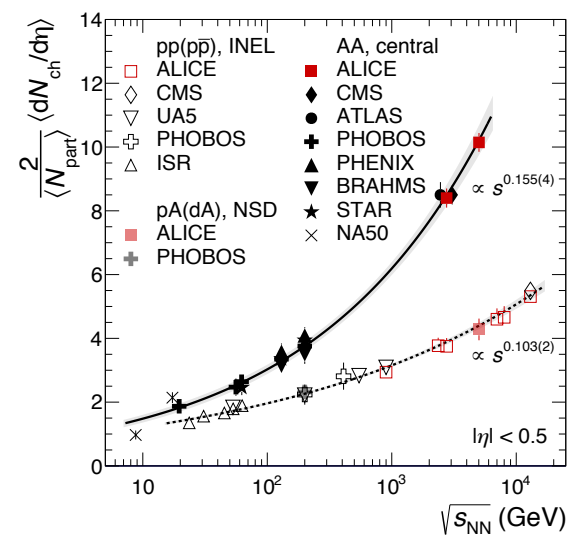

A

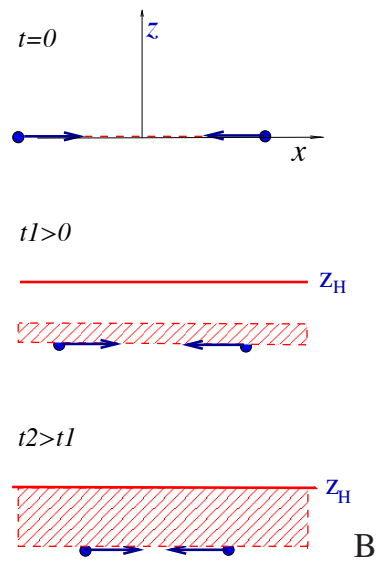

Figure 2. A. The plot of the total number of charged particles versus energy $\sqrt{s}$ for protons and $\sqrt{s_{N N}}$ per pair of nucleons for ions collisions. Plot from ALICE collaboration [13]. B. The schematic cartoon of the black hole formation in the bulk after collision of particles on the boundary.

In [26] it has been shown that the model that reproduces the Cornell potential also gives a correct energy dependence of multiplicities if we assume that the multiplicity is related with the dual entropy produced during a limited time period. However in this consideration there is a limitation on the possible energy of colliding shock walls[26]. Since in this consideration we have used a more or less general isotropic background reproducing AdS at UV and confinement at IR, we can think that the assumption about the isotropic background prevent to reproduce (3) at hight energy.

In [30] we have considered a special anisotropic backgrounds, parametrized by a paranmeter $v$ and have got

$$
\mathcal{M}_{v} \sim s^{1 /(v+2)}
$$

and therefore to get an estimation (3) we take $v=4.45$. Note, that $v=4$ gives $\mathcal{M}_{v=4} \sim s^{0.167}$, that is rather closed to (3). $v=1$ corresponds to the AdS case.

Let us note, that the idea to use anisotropic background in the holographic approach to QGP is not new. There were several proposals to deal with anisotropic backgrounds, see for example [35] and refs therein. Up to couple years ego, it has been believed that the matter was in the pre-equilibrium period up to $1 \mathrm{fm} / \mathrm{c}$ and then the QGP appears and it is isotropic. However now there is a believe that the QGP is created after a very short time after collision, $\tau_{\text {therm }} \sim 0.1 \mathrm{fm} / \mathrm{c}$, and it is anisotropic ("anisotropic" means a spatially anisotropy) for a short time $\tau$ after collision, $0<\tau_{\text {therm }}<\tau<\tau_{\text {iso }}$, and the time of locally isotropization is about $\tau_{\text {iso }} \sim 2 \mathrm{fm} / \mathrm{c}$ [36]. 


\section{Holographic Thermalization}

\subsection{Thermalization}

Suppose we deal with a correlator of a time depending quantity $O(t),\langle O(t)\rangle$. If at large $t$ the system exhibits the behaviour

$$
<O(t)>\underset{t \rightarrow \infty}{\rightarrow}<<O>>
$$

where

$$
<<O>>=\operatorname{tr}\left(e^{-\beta H} O\right)
$$

one says that the system goes to the stationary state and this state is a thermal state, or in other words the system thermalizes. The simples thermalization process can be described by interaction with thermostat. For one oscillator interacting with thermostat one can show the thermalization explicitly.

\subsection{Thermalalization as a black hole creation in AdS}

According the holographic scenario the thermalization process in d-dimensional space-time can be understood as horizon formation in a $\mathrm{d}+1$ dimensional gravitational theory. To initiate the process of $\mathrm{BH}$ formation one has to perturb the initial AdS metric. According to AdS/CFT correspondence this deformation should be related with the deformation of the energy-momentum tensor of the boundary theory. So the idea is to make some perturbation of AdS metric that near the boundary mimics the heavy ions collisions and see what happens, see Fig.2.B.

One can consider several deformations of AdS metric:

- a deformation by adding colliding gravitational shock waves.

- we can drop of a shell of matter with vanishing rest mass ("null dust"),

- we can study the toy 3-dim model with colliding ultra relativistic particles

It seems natural to think about an ultrarelativistic nucleus as a shock wave in 4-dimensional spacetime. One can also assume that this shock has a profile function. According AdS CFT correspondence to this energy-momentum tensor corresponds the metric of a shock wave in $\mathrm{AdS}_{5}$ of the following form

$$
d s^{2}=L^{2} \frac{-d u d v+d x_{\perp}^{2}+\phi\left(x_{\perp}, z, L\right) \delta(u) d u^{2}+d z^{2}}{z^{2}},
$$

here $u, v$ are light-cone variables, $u=x_{0}+x_{\|}, v=x_{0}-x_{\|}, \phi\left(x_{\perp}, z, L\right)$ is the profile function, $L$ is the scale parameter. For $\phi\left(x_{\perp}, z, L\right)=0$ the metric (7) is nothing but the Poincare metric for $\mathrm{AdS}_{5}$. For two colliding nuclei corresponds the following metric in $\mathrm{AdS}_{5}$

$$
d s^{2}=L^{2} \frac{-d u d v+d x_{\perp}^{2}+\phi_{1}\left(x_{\perp}, z, L\right) \delta(u) d u^{2}+\phi_{2}\left(x_{\perp}, z, L\right) \delta(v) d v^{2}+d z^{2}}{z^{2}} .
$$

It is interesting to note that gaussian regularization of the point shock wave profile function $\phi\left(x_{\perp}, 0\right)$ exactly corresponds to the Woods-Saxon profile for the nuclear density. This correspondence give us L equal to 4.3 fermi for gold and L equal to 4.4 fermi for lead.

It is natural to ask the question: can we guaranty the black hole formation under collisions of these shock waves. If the answer is "yes", then I'll take the entropy of this formed black hole as a multiplicity of particles production. This idea has been explored in several papers [17-24]. The idea to relate multiplicities with the thermodynamic characteristic of the media produced as a result of a collision of particles comes back to Landau and Fermi [27-29]. 


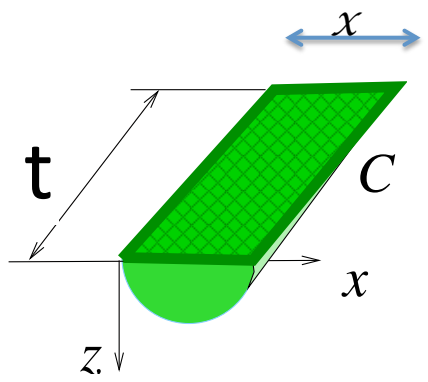

$t>x$

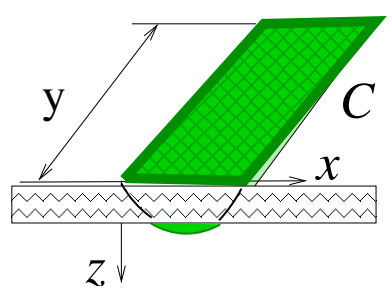

B

A

Figure 3. A. Wilson loop in the static background. B. Wilson loop in the Vaidya background

\subsection{Thermalization time}

Thermalization time depends on the physical quantities that expect to be thermalized. This issue has been discuss in details in our talk [37] and is not discussed here, see also [38-40] for estimations of thermalization time of different variables in the Lifshitz-type background.

\section{Physics in the Lifshitz-type background}

\subsection{Lifshitz-type background}

In [30] we have studied collisions of shock waves in the anisotropic background

$$
d s^{2}=2 \pi \alpha^{\prime}\left(\frac{-d t^{2}+d x^{2}}{z^{2}}+\frac{d y_{1}^{2}+d y_{2}^{2}}{z^{2 / v}}+\frac{d z^{2}}{z^{2}}\right)
$$

where $v$ is the critical exponent.

After collision the black hole is produced and the stationary black hole metric in this background is [31]

$$
d s^{2}=z^{-2}\left(-f(z) d t^{2}+d x^{2}\right)+z^{-2 / v}\left(d y_{1}^{2}+d y_{2}^{2}\right)+\frac{d z^{2}}{z^{2} f(z)}
$$

where the blackening function is

$$
f=1-m z^{2 / v+2} \text {. }
$$

Motivated by [32, 33] we deform this background as ${ }^{1}$

$$
d s^{2}=\frac{b(z)}{z^{2}}\left(\left(-f(z) d t^{2}+d x^{2}\right)+z^{2-2 / v}\left(d y_{1}^{2}+d y_{2}^{2}\right)+\frac{d z^{2}}{f(z)}\right)
$$

where the blackening function takes into account a non-zero chemical potential

$$
f=1-\left(\frac{1}{z_{h}^{2 / v+2}}+q^{2} z_{h}^{2}\right) z^{2 / v+2}+q^{2} z^{2 / v+4}
$$

\footnotetext{
${ }^{1}$ The problem of finding of shock waves in the background (12) and estimation of the trapped surface in this background has not solved yet, compare with [22, 34]
} 
and $b(z)$ is a factor that provides the quark confinement in the isotropic case [32]

$$
b(z)=\exp \left(\frac{c z^{2}}{2}\right)
$$

and the zero-component of the vector field is given by

$$
A_{0}(z)=i\left(\mu-\frac{4 \sqrt{3} q}{c}\left(1-e^{-c z^{2} / 4}\right)\right) .
$$

According the AdS/CFT dictionary, the boundary value $A_{0}(0)$ is related to the quark chemical potential:

$$
A_{0}(0)=i \mu
$$

The vanishing of $A_{0}(z)$ at the horizon, $A_{0}\left(z_{h}\right)=0$, sets a relation between the chemical potential and the charge of the black hole:

$$
\mu=\frac{\sqrt{3} q}{c}\left(1-e^{-c z_{h}^{2} / 4}\right)
$$

The temperature $T$ is defined by the relation

$$
T=\frac{1}{4 \pi}\left|\frac{d f}{d z}\right|_{z=z_{h}}=\frac{1}{2 \pi} \frac{\frac{1}{v}+1}{z_{h}}\left(1-\frac{Q_{v}^{2}}{2}\right),
$$

where $Q_{v}=\sqrt{\frac{2}{\frac{1}{v}+1}} q z_{h}^{\frac{1}{v}+2}$. We can also present the dependence of $T$ on the chemical potential

$$
T=\frac{\left(\frac{1}{v}+1\right)}{2 \pi z_{h}}\left(1-\frac{c^{2} \mu^{2} z_{h}^{\frac{2}{v}+4}}{3\left(\frac{1}{v}+1\right)\left(1-e^{-\frac{c z_{h}^{2}}{2}}\right)^{2}}\right)
$$

see Fig.4.

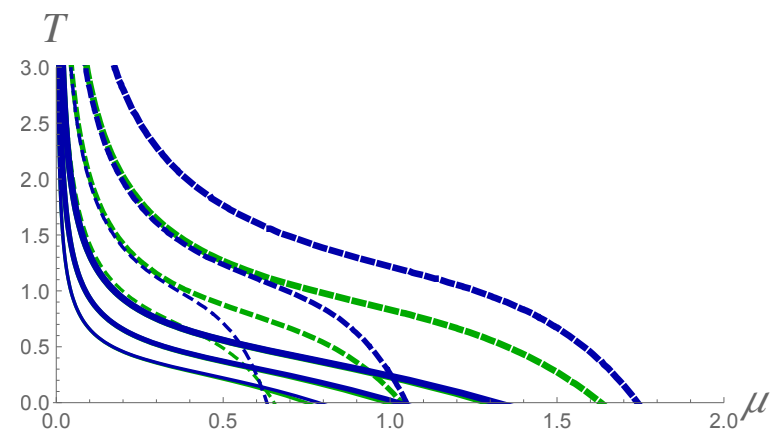

Figure 4. Relation between $T$ and $\mu$ given by formula (18) and (17) for isotropic (solid lines) and anisotropic case with parameter $v=4$ (dashed lines) for different values of the charge $q: q=2$ (solid/dashed thick line), $q=1$ (solid/dashed line) and $q=0.5$ (thin solid/dashed line) and different confinement factors: $c=0.2$ (blue lines) and $c=0.3$ (green lines) 


\subsection{Temporal Wilson loop in the charged quark confinement background}

\subsubsection{Energy between quarks located along $x$-direction in the quark confinement background}

The potential of the interquark interaction along $\mathrm{x}$-direction can be extracted from the rectangular time-like Wilson loop of size $T \times X$, i.e. the loop in which one side is infinite along the time direction, and the other is along the $x$ direction

$$
W(T, X)=\left\langle\operatorname{Tr}_{F} e^{i \oint_{I \times X} d x_{\mu} A_{\mu}}\right\rangle \sim e^{-V(X) T},
$$

$F$ means the fundamental representation. Following the holographic approach [41-43] the expectation value of the Wilson loop in the fundamental representation calculated on the gravity side reads as:

$$
W[C]=e^{-S_{(x t)}},
$$

where $C=T \times X$ in a contour on the boundary, $S_{x t}$ is the minimal Nambu-Goto action of the string hanging from the contour $C$ in the bulk

$$
S_{x t}=\frac{1}{2 \pi \alpha^{\prime}} \int d \sigma^{1} d \sigma^{2} \sqrt{-\operatorname{det}\left(h_{\alpha \beta}\right)}
$$

where $h_{\alpha \beta}$ is the induced metric of the world-sheet $h_{\alpha \beta}=g_{M N} \partial_{\alpha} X^{M} \partial_{\beta} X^{N}, \alpha, \beta=0,1, g_{M N}$ is the background metric, $M, N=0, \ldots, 4, X^{M}=X^{M}\left(\sigma^{0}, \sigma^{1}\right)$ specify the string worldsheet with the worldsheet coordinates $\sigma^{1}, \sigma^{2}$. We parametrize the worldsheet as $X^{0} \equiv t=\sigma^{0}$ and $X^{1} \equiv x=\sigma^{1}$ and consider the static configuration $X^{4} \equiv z=z(x)$. The action $S_{(x t)}$ is:

$$
S_{x t}=\frac{T}{2 \pi \alpha^{\prime}} \int \frac{b(z)}{z^{2}} \sqrt{f(z)+z^{\prime 2}} d x .
$$

where ' means $\frac{d}{d x}$. If we take $z^{\prime}=0$ in (23) we get the "potential"

$$
V_{x}(z)=\frac{b(z)}{z^{2}} \sqrt{f(z)}
$$

We consider the symmetrical parametrization $z( \pm \ell)=0$ and $z(0)=z_{*}, z^{\prime}(0)=0$. The distance between the two endpoints of the string can be represented as

$$
L_{x}=2 \int_{\infty}^{z_{*}} \frac{d z}{z^{\prime}}=2 \int_{0}^{z_{*}} \frac{d z}{\sqrt{f(z)} \sqrt{\left(\frac{V_{x}^{2}(z)}{V_{x}^{2}\left(z_{*}\right)}-1\right)}}
$$

We consider the case when $z_{*}<z_{\left.h_{i}\right)}$, where $z_{h_{i_{0}}}$ is the smallest of the horizons, i.e. if $f\left(z_{h_{i}}\right)=0$, then $z_{h_{i}}<z_{h_{i}}$. Therefore $f(z)>0$ for $0<z<z_{*}$.

We get the energy of the string we subtract the mass of the two free quark [35, 42, 43]

$$
\pi \alpha^{\prime} E_{x}=\int_{0}^{z_{*}} \frac{d z}{z^{2}}\left[\frac{b(z) V(z)}{\sqrt{V^{2}(z)-V^{2}\left(z_{*}\right)}}-1\right]-\frac{1}{z_{*}}+m^{\frac{v}{2 v+2}} e^{c m^{-\frac{2 v}{2 v+2}}}-\sqrt{\pi c} \operatorname{erfi}\left(\sqrt{c} m^{-\frac{v}{2 v+2}}\right) .
$$

To guaranty that the expression under the second square root in (25) is positivity we have to integrate along the curve where the potential $V_{x}(z)$ is a decreasing function. Depending on the parameters $c, z_{h}, v$ and $q$ the function $V_{x}(z)$ can have two extremal points, one minimum and one maximum, or have no extremal point at all at the interval $0<z<z_{h_{i_{0}}}$, see Fig. 5 and Fig. 6. 


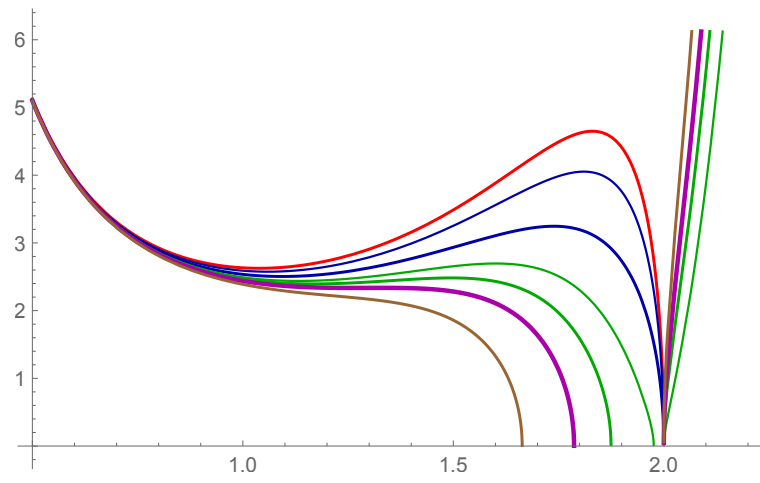

Figure 5. The potential $V(z)=V\left(z, c, q, v, z_{h}\right)$ given by (24) with $b(z)$ given by (14) with $c=2$ and $f(z)=f\left(z, q, v, z_{h}\right)$ given by (13) with $v=1$ and $z_{h}=2$. The red line shows $V(z)$ for $q=0$. We see that the corresponding potential has minimum and maximum. The blue lines show $V(z)$ for $q=0.1$ and $q=0.15$. The corresponding potential have minimum and maximum. The green lines show $V(z)$ for $q=0.18$ and $q=0.195$. The corresponding potential have minimum and maximum and the second zero of the corresponding function $f(z)=f\left(z, q, 1, z_{h}\right)$ are on the left of $z_{h}=2$. The magenta line show $V(z)$ for $q=0.21$. The corresponding potential has no local extremum and the second zero of $f(z)$ is at $z_{h_{2}}=1.784$. For $q=0.21$ (the brown line) the corresponding potential has no minimum and maximum on $0<z<z_{h_{2}}$ and the potential is the decreasing function at $z<z_{h_{2}}=1.662$

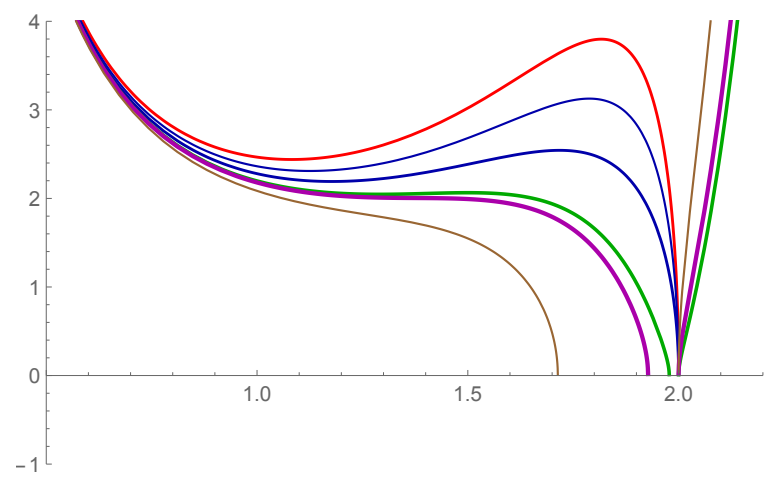

Figure 6. The potential $V_{x}(z)=V\left(z, c, q, v, z_{h}\right)$ given by (24) with $b(z)$ given by (14) with $c=2$ and $f(z)=$ $f\left(z, q, v, z_{h}\right)$ given by (13) with $v=4$ and $z_{h}=2$. The red line shows $V(z)$ for $q=0$. We see that the corresponding potential has minimum and maximum. The blue lines show $V(z)$ for $q=0.15,0.2$. The corresponding potential have minimum and maximum. The green line shows $V(z)$ for $q=0.238$. The second zeros of the corresponding function $f(z)$ at $z=1.97$. The magenta line shows $V(z)$ for $q=0.245$. The corresponding potential has no minimum and maximum and the second zero of the corresponding function $f(z)=f\left(z, q, 1, z_{h}\right)$ is at $z_{h_{2}}=1.893$. The brown line shows $V(z)$ for $q=0.28$ and the corresponding potential has no minimum and maximum on $0<z<z_{h_{2}}$, where $z_{h_{2}}=1.71$ is the second zero of the corresponding functions $f(z)$. The corresponding potential (the brown lines) are the decreasing functions. The critical charge $\left.q_{c r}\right|_{c=2, z_{h}=2}=0.245$ 
Therefore, as for isotropic cases [44-50] ${ }^{2}$, we have two cases:

- There is no extremal point in the interval $0<z<z_{h_{0}}$. This case corresponds to the deconfinement phase and dependence of $\ell$ on $z_{*}$ has the form as presented in Fig.7.a). We see that there are two branches indicated by blue and red color, the same $\ell$ can be obtained by two different $z_{*}$, except $z_{*}=z_{* 0}$ where $\ell$ reaches its maximal value $\ell_{0}$, i.e. $\ell=\ell\left(z_{*}\right)$ monotonically increases from $\ell(0)=0$ to $\ell\left(z_{*_{0}}\right)=\ell_{0}$ (the first branch shown by the blue color) and $\ell=\ell\left(z_{*}\right)$ monotonically decreases from $\ell\left(z_{*_{0}}\right)=\ell_{0}$ to $\ell\left(z_{h_{0}}\right)=0$ (the second branch). In Fig.7.a) $z_{h_{0}}=z_{h}$. The plot in Fig.7.b) shows the values of the energy between quarks located along $\mathrm{x}$-direction as function of $z_{*}$ for two branches. The corresponding values of the energy as functions $\ell$ are presented in Fig.7.c). As shown in this plot, the values of energy corresponding to the second branch are large than those for the first one.

$L$

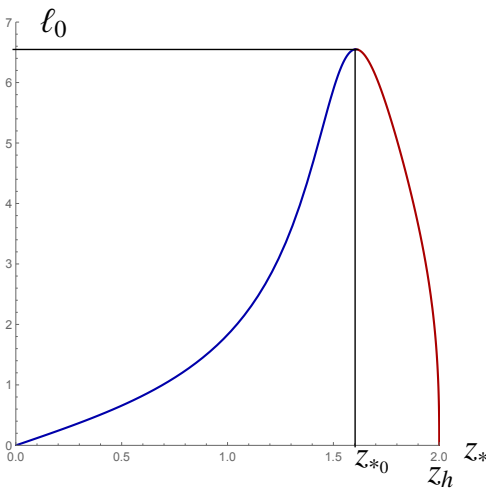

a)
$E_{x}$

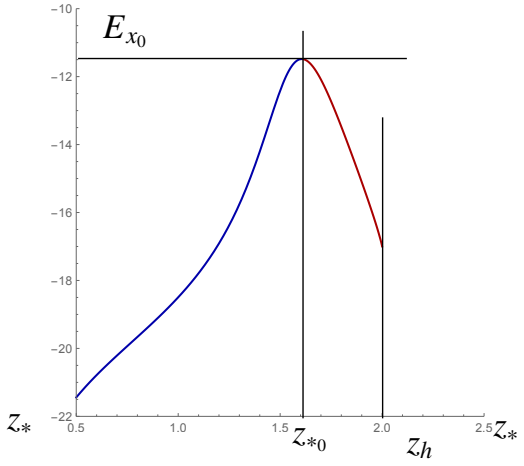

$E_{x}$

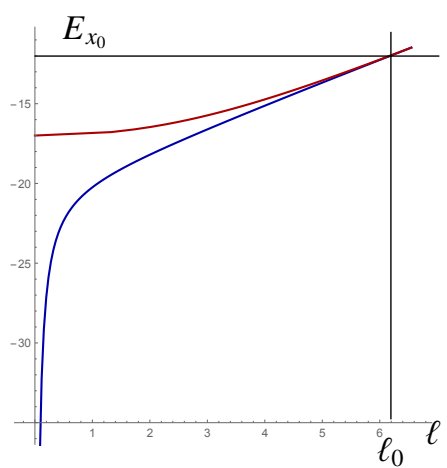

b)

c)

Figure 7. Above the critical point (deconfinment): a) $L=L\left(z_{*}\right)$, b) $E_{x}=E_{x}\left(z_{*}\right)$ and c) $E_{x}=E_{x}(\ell)$. Here we put $\alpha^{\prime}=1 / \pi$

- There are two extremal points in the interval $0<z<z_{h_{i_{0}}}, z_{\min }=z_{0}$ and $z_{\max }=z_{0}^{\prime}$ and the potential is decreasing function only on the intervals

$$
0<z<z_{\min } \text { and } z_{\max }<z<z_{h_{i_{0}}}
$$

we can guarantee that $V(z) / V\left(z_{*}\right)<1$ only in the region (27).

This case corresponds to the confinement phase and the dependence of $L$ on $z_{*}$ is presented in Fig.8.a). Suppose that $V(z)$ has a local minimum at $z=z_{0}$. In this case the potential $V_{x}(z)$ is a decreasing function from $z=0$ up to $z=z_{0}$. The first branch $L=L(z)$ is shown by the blue line in the plot in Fig.8.a). and $L(z) \rightarrow \infty$ when $z \rightarrow z_{0}-0$. For $z_{0}<z<z_{0}^{\prime}$ gets $L=L(z)$ an imaginary part and at $z=z_{0}^{\prime}$ starts the second branch. For the second branch $E_{2}<E(\ell)<E_{2}^{\prime}$.

In Fig. 9 we present the same quantities as inFig.7 and Fig. 8 at the same parameters except that in Fig.7 and Fig. $8 v=1$ (isotropic case) and in Fig.9 $v=4$ (anisotropic case, longitudinal orientation of the Wilson loop). The confinement solution is realized on the first branch discussed above. Let us estimate the string tension for this configuration. Suppose that

$$
\left.V^{\prime}(z)\right|_{z=z_{0}}=0, \quad V^{\prime \prime}\left(z_{0}\right)>0
$$

\footnotetext{
${ }^{2}$ Note, that the isotropic case with the confinement factor has been studied at zero chemical potential in [44, 45] and with nonzero chemical potential in [46-50]
} 

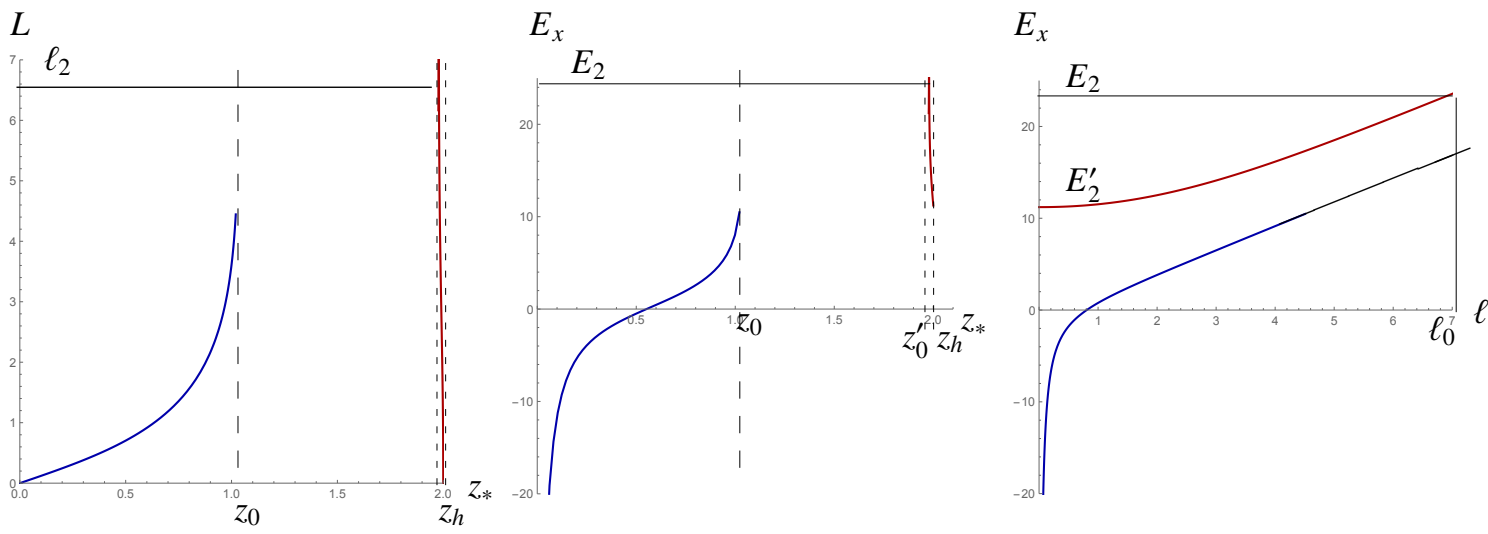

a)

b)

c)

Figure 8. Below the critical point (confinment): a) $L=L\left(z_{*}\right)$, b) $E_{x}=E_{x}\left(z_{*}\right)$ (not that in this case we make a different subtraction) and c) $E_{x}=E_{x}(\ell)$

Near $z=z_{0}$ we have $V^{\prime \prime}\left(z_{0}\right)>0$ and

$$
\begin{aligned}
& \sqrt{\frac{V(z)^{2}}{V\left(z_{0}\right)}-1}=\sqrt{\frac{V^{\prime \prime}\left(z_{0}\right)}{V\left(z_{0}\right)}}\left(z-z_{0}\right)+O\left(z-z_{0}\right)^{2} \\
& L_{1} \underset{\substack{z_{*} \sim z_{0} \\
\sim}}{\sim}-2 \sqrt{\frac{V\left(z_{0}\right)}{f\left(z_{0}\right) V^{\prime \prime}\left(z_{0}\right)}} \ln \left(z_{0}-z_{*}\right)
\end{aligned}
$$

We can also calculate the energy near $z_{*}=z_{0}$ using (26). We have

$$
S_{x t} \underset{z_{*} \sim z_{0}}{\sim}-\frac{T}{2 \pi \alpha^{\prime}} \frac{b\left(z_{0}\right)}{z_{0}^{2}} \frac{1}{\sqrt{\frac{V^{\prime \prime}\left(z_{0}\right)}{V^{\prime}\left(z_{0}\right)}}} \ln \left(z_{0}-z_{*}\right)
$$

Therefore we get

$$
S_{x t} \underset{L \rightarrow \infty}{\sim} \frac{T}{\pi \alpha^{\prime}} \frac{L_{1}}{2} \frac{b\left(z_{0}\right) \sqrt{f\left(z_{0}\right)}}{z_{0}^{2}}
$$

and

$$
\sigma_{x}=\frac{V_{x}\left(z_{0}\right)}{2 \pi \alpha^{\prime}}
$$

\subsubsection{Energy between quarks located along y-direction in the quark confinement background} (12)

The Nambu-Goto action $S_{y t}$ of the string hanging from the contour $C$, being the rectangular with sites along the time direction and one of transversal directions, in the bulk is

$$
S_{y t}=\frac{T i}{2 \pi \alpha^{\prime}} \int \frac{b(z)}{z^{2}} \sqrt{z^{2-2 / v} f(z)+z^{\prime 2}} d x .
$$



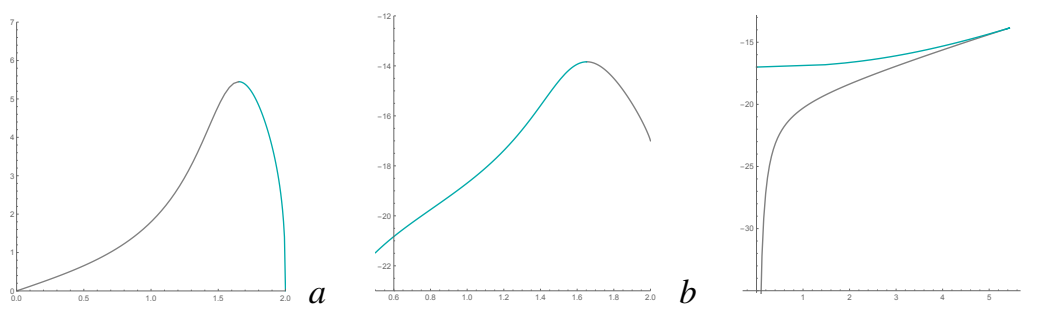

$c$
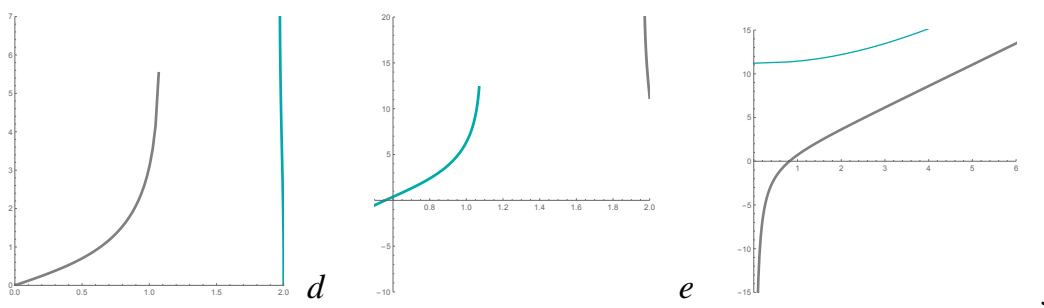

Figure 9. The same quantities as in Fig. 8 and 8 at the same values of parameters, except that now we deal with the anisotropic case, $v=4$. a),b) and c) above the critical point (deconfinment): a) $L=L\left(z_{*}\right)$, b) $W=W\left(z_{*}\right)$ and c) $W=W(L)$. d), e) and f) above the critical point (deconfinment): d) $L=L\left(z_{*}\right)$, e) $W=W\left(z_{*}\right)$ (note that in this case we make a different subtraction) and f) $W=W(L)$.

The corresponding "potential" is, see Fig.10,

$$
V_{y}(z)=\frac{b(z) \sqrt{f(z)}}{z^{1 / v+1}}
$$

and for $L_{y}$ we have

$$
L_{y}=2 \int_{0}^{z_{*}} \frac{z^{1 / v-1} d z}{\sqrt{f(z)} \sqrt{\frac{V^{2}(z)}{V^{2}\left(z_{*}\right)}-1}} .
$$

To get for the energy of the string between two quarks stretched in the $y$-direction we perform the subtraction of the two quark mass that corresponds the subtraction of the action on the sheet starting at the boundary $z=0$ and ending at the horizon

$$
\pi \alpha^{\prime} E_{y}=\int_{0}^{z_{*}} \frac{d z}{z^{2}}\left[\frac{b(z) V(z)}{\sqrt{V^{2}(z)-V^{2}\left(z_{*}\right)}}-1\right]-\frac{1}{z_{*}}+m^{\frac{v}{2 v+2}} e^{c m^{-\frac{2 v}{2 v+2}}}-\sqrt{\pi c} \operatorname{erfi}\left(\sqrt{c} m^{-\frac{v}{2 v+2}}\right) .
$$

Now, suppose that $V_{y}(z)$ has a minimum at $z=z_{0}$, we can estimate the contributions near this minimum, we have

$$
\begin{aligned}
\pi \alpha^{\prime} E_{y} \underset{z_{*} \sim z_{0}}{\sim} \frac{\ln \left(z_{0}-z_{*}\right)}{z_{0}^{2}} \frac{b\left(z_{0}\right)}{\sqrt{\frac{V^{\prime \prime}\left(z_{0}\right)}{V\left(z_{0}\right)}}} \\
L_{y} \underset{z_{*} \sim z_{0}}{\sim} \frac{z_{0}^{1 / v-1} \ln \left(z_{0}-z_{*}\right)}{\sqrt{f\left(z_{0}\right)}} \frac{1}{\sqrt{\frac{V^{\prime \prime}\left(z_{0}\right)}{V\left(z_{0}\right)}}}
\end{aligned}
$$




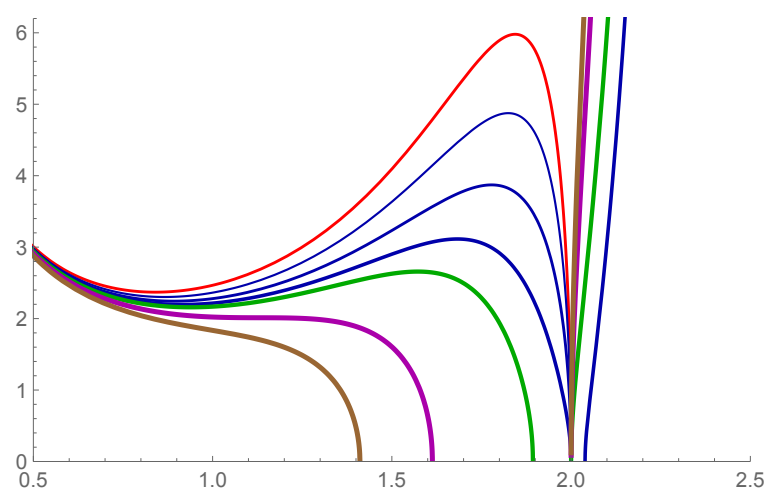

Figure 10. The potential $V_{y}(z)=V\left(z, c, q, v, z_{h}\right)$ given by (34) with $b(z)$ given by (14) with $c=2$ and $f(z)=f\left(z, q, v, z_{h}\right)$ given by (13) with $v=4$ and $z_{h}=2$. The red line shows $V_{y}(z)$ for $q=0$. We see that the corresponding potential has minimum and maximum. The blue lines show $V_{y}(z)$ for $q=0.15,0.2$ and $q=0.23$. The corresponding potentials have minimum and maximum. The green line shows $V_{y}(z)$ for $q=0.25$ and second zeros of the corresponding function $f(z)$ is at $z_{h_{2}}=1.89$. The magenta line show $V(z)$ for $q=0.3$. The corresponding potential has no minimum and maximum and the second zero of the corresponding function $f(z)=f\left(z, q, 1, z_{h}\right)$ is at $z_{h_{2}}=1.61$. The brown line shows $V_{y}(z)$ for $q=0.35$ and the corresponding potential have no minimum and maximum on $0<z<z_{h_{2}}$, where $z_{h_{2}}=1.40$ is the second zero of the corresponding functions $f(z)$. The critical charge $\left.q_{c r}\right|_{c=2, z_{h}=2}=0.3$

Therefore,

$$
\frac{\pi \alpha^{\prime} S_{y t}}{T}=L_{y} V\left(z_{0}\right)
$$

and

$$
\sigma_{y}=\frac{V_{y}\left(z_{0}\right)}{2 \pi \alpha^{\prime}}
$$

i.e. we get the answer similar to (32).

In Fig. 11 we present the same quantities as inFig.7 and Fig. 8 at the same parameters except that in Fig. 7 and Fig. $8 v=1$ (isotropic case) and in Fig.11 $v=4$ (anisotropic case, transversal orientation of the Wilson loop). We see that for the transversal orientation at the same parameters as for the isotropic case we can get different solutions: for the isotropic case, as well as for the anisotropic longitudinal case, $c=1.2$ corresponds to the deconfined phase, meanwhile this value of $c$ in the anisotropic transversal case case corresponds to the confined phase. This observation indicates that the phase diagram should be essential depends on the orientation in the case of the anisotropic background.

\subsection{Holographic anisotropic QCD phase diagrams}

In Fig. 12 we present the phase diagram for isotropic and anisotropic $E_{x}$ and $E_{y}$ cases. Holographic isotropic QCD phase diagram has been studied previously in bottom-up approaches [46-48]. We see that for zero chemical potential the deconfinement occurs for the low temperature in the anisotropic case, and for zero temperature the deconfinement phase survives for the large chemical potential in the anisotropic case as well. 

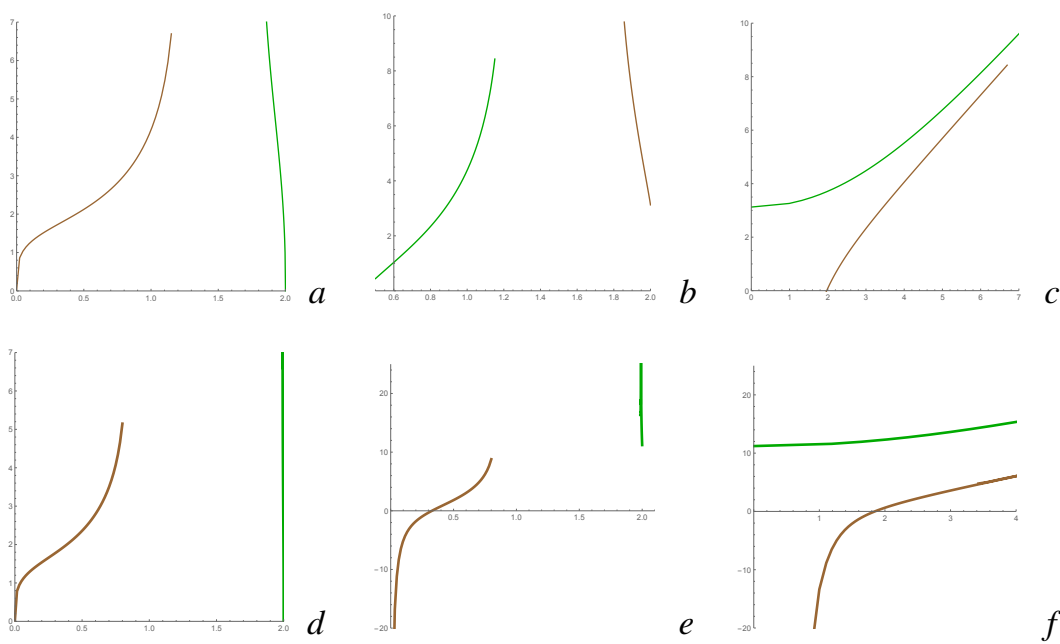

Figure 11. The same quantities at the same parameters as in Fig.9 except that now we deal with the transversal orientation of the Wilson loop, $v=4$. We see that both values of $c, c=1.2$ and $c=2$, correspond to the confinement phase: a) and d) $L=L\left(z_{s}\right)$, b) and e) $W=W\left(z_{s}\right)$ (note that in this case we make a different subtraction), c) and f) $W=W(L)$.

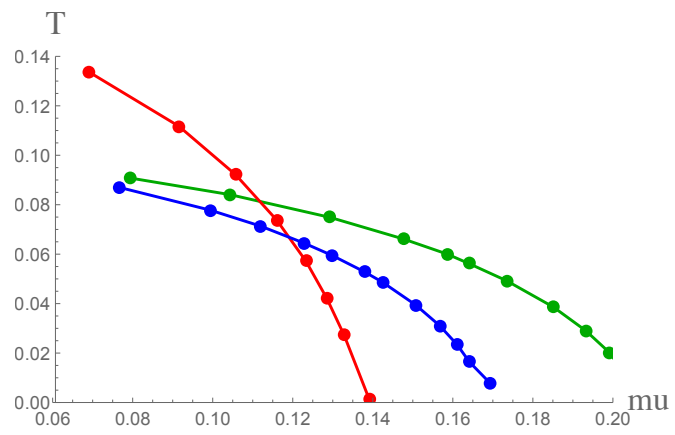

Figure 12. The deconfinement transition line in the $(\mu, T)$ plane. Phase transitions lines dividing the plane in two regions, a hadron phase near the origin, and a deconfined phase beyond the curve. The red line(points) corresponds to the isotropic case, the blue line (points) corresponds to the $E_{x}$ in the anisotropic case with $v=4$, the green to $E_{y}$ in the same anisotropic case. The dots represent numerical data points, while the solid interpolating lines are included "by hands"

Fig.12 shows the dependence of the transition line on the orientation. Since quarks can be arbitrary oriented in respect to the collision line, this dependence on the orientation leads to a broadening of the line separating confinement and deconfinement phases in the $(\mu, T)$-plane. Decreasing of anisotropy decreases the broadening of the phase transition boundary.

Let us remind that in isotropic case the confinement/deconfinment diagram has been studied in lattice QCD. Experimentally the phase boundary between hadronic matter and the quark - gluon plasma in relativistic heavy ion collisions is probing using the HRM [51, 52] 


\section{Direct photons and electric conductivity}

The thermal-photon production from the QGP plays an essential role, since photons after they are produced in HIC almost do not interact with the QGP and, therefore, they give us the local information in heavy ion collisions. The photon-emission rate is related to the retarded correlator of currents in momentum space [53]

$$
G_{\mu \nu}^{R}(k)=i \int d^{4}(x-y) e^{i k \cdot(x-y)} \theta\left(x^{0}\right)\left\langle\left[J_{\mu}^{a}(x), J_{v}^{b}(0)\right]\right\rangle,
$$

so that in the thermal equilibrium it is given by the light-like retarded correlator as

$$
d \Gamma=-\frac{d^{3} k}{(2 \pi)^{3}} \frac{e^{2} n_{b}(|\mathbf{k}|)}{|\mathbf{k}|} \operatorname{Im}\left[\operatorname{tr}\left(\eta^{\mu v} G_{\mu \nu}^{a b R}\right)\right]_{k^{0}=|\mathbf{k}|},
$$

where $\Gamma$ denotes the number of photons emitted per unit time per unit volume and $n_{b}(|\mathbf{k}|)$ denotes the thermal distribution function for bosons.

The imaginary part of the retarded correlator is also related with the spectral function

$$
\chi_{\mu \nu}(k)=-2 \mathfrak{J} G_{\mu \nu}^{R}(k)
$$

and due to the Kubo formula with the conductivity tensor

$$
\sigma_{\mu \nu}(k)=-\frac{G_{\mu \nu}^{R}(k)}{i w}
$$

The spectral function can be evaluated by holography using the flow method related with the membrane paradigm [54]. Below we shortly remind this prescription, see also [55, 56]. One starts from the Maxwell action in the given background

$$
S_{M}=-\mathcal{N} \int d^{5} x \sqrt{-g} \frac{V_{d i l}(\phi)}{4} F^{M N} F_{M N},
$$

where it is assumed also the presence of the dilaton field $\phi$, and denote by $\mathcal{N}$ the normalization constant including the 5-dimensional gravitational constant $G_{5}$ etc.

The gauge field $A_{\mu}(t, \mathbf{x}, r)$ it is taken to be

$$
A_{\mu}(t, \mathbf{x}, z)=\int \frac{d^{4} k}{(2 \pi)^{4}} e^{-i \omega t+i \mathbf{k} \mathbf{x}} \mathcal{A}_{\mu}(z, \omega, k), \quad \mathcal{A}_{\mu}(z, \omega, k)=A_{\mu}(z, \omega, k) a_{\mu}(\omega, k)
$$

$A_{\mu}(t, \mathbf{x}, z)$ satisfies the Maxwell equation, and $A_{\mu}(r, \omega, k)$ satisfies the boundary conditions at the boundary

$$
\lim _{z \rightarrow 0} A_{\mu}(z, \omega, k)=1
$$

and the infalling boundary conditions at the horizon,

$$
\lim _{z \rightarrow z_{h}} A_{\mu}(z, \omega, k)=0
$$

One can consider for simplicity the case $k=k_{x}, k_{y_{1}}=k_{y_{2}}=0$.

Let us parametrize the metric as

$$
d s^{2}=\frac{b^{2}(z)}{z^{2}}\left(-f(z) d t^{2}+d x^{2}+p(z)\left(d y_{1}^{2}+d y_{2}^{2}\right)+\frac{d z^{2}}{f(z)}\right),
$$


In our model $p(z)=z^{2-2 / v}$. The Maxwell action (with the dilaton) has the form

$$
\int d^{4} x d z V(z)\left(-\frac{F_{01}^{2}+F_{02}^{2}}{f p}-\frac{F_{03}^{2}}{f}+f\left(\frac{F_{1 r}^{2}+F_{2 r}^{2}}{p}+F_{3 r}^{2}\right)-F_{0 r}^{2}+\frac{F_{12}^{2}}{p^{2}}+\frac{F_{13}^{2}+F_{23}^{2}}{p}\right)
$$

here

$$
V(z)=V_{d i l}(\phi(z)) \frac{b(z)}{z}
$$

and

$$
F_{M N}=\partial_{M} A_{N}-\partial_{N} A_{M}
$$

Assume that the fluctuating vector field depends only on $x_{0}, x_{3}, r$-coordinates, one can write the E.O.M. in term of $E_{L}$ and $E_{\perp}$

$$
\begin{aligned}
& E_{L}^{\prime \prime}+\left(\frac{f^{\prime}}{f} \frac{w^{2}}{w^{2}-f k^{2}}+\frac{V^{\prime}}{V}\right) E_{L}^{\prime}+\frac{w^{2}-f k^{2}}{f^{2}} E_{L}=0 \\
& E_{\perp, i}^{\prime \prime}+\left(-\frac{p^{\prime}}{p}+\frac{V^{\prime}}{V}+\frac{f^{\prime}}{f}\right) E_{\perp, i}^{\prime}+\frac{w^{2}-k^{2}}{f} E_{\perp, i}=0, \quad i=1,2
\end{aligned}
$$

here $E_{L}=k A_{0}+w A_{3}$ and $E_{\perp, i}=w A_{\perp, i}, i=1,2$.

The boundary terms come from that terms the total action (50) which have the z-derivative and we get

$$
S_{\text {boundary }}=S_{\text {boundary }, 1}+S_{\text {boundary }, 2}
$$

where $S_{\text {boundary, } 1}$ and $S_{\text {boundary, } 2}$ are

$$
\begin{aligned}
S_{\text {boundary }, 1} & =\int d^{4} x \frac{f}{w^{2}-k^{2} f} E_{L} E_{L}^{\prime} \\
S_{\text {boundary }, 2} & =\int d^{4} x V \frac{f}{p w^{2}} E_{\perp} E_{\perp}^{\prime}, \quad E_{\perp} E_{\perp}^{\prime} \equiv \sum_{i=1,2} E_{\perp, i} E_{\perp, i}^{\prime}
\end{aligned}
$$

In the analogy with the isotropic case we introduce

$$
\begin{aligned}
& \zeta_{\perp}=-\frac{V f}{p w} \frac{\partial_{r} E_{i}^{\perp}}{E_{i}^{\perp}}, \\
& \zeta_{L}=-\frac{V f}{\omega} \frac{\partial_{r} E_{L}}{E_{L}},
\end{aligned}
$$

which satisfy the following equations

$$
\begin{aligned}
\zeta_{\perp}^{\prime}-\frac{w}{f}\left[\frac{p}{V} \zeta_{\perp}^{2}+\frac{V}{p}\left(1-f \frac{k^{2}}{w^{2}}\right)\right] & =0 . \\
\zeta_{L}^{\prime}+\frac{k^{2} f^{\prime}}{w^{2}-f k^{2}} \zeta_{L}-\frac{w}{f}\left[\frac{\zeta_{L}^{2}}{V}+V\left(1-f \frac{k^{2}}{w^{2}}\right)\right] & =0
\end{aligned}
$$

From these eqs follows that if $\zeta_{\perp}^{\prime} \neq \infty$ and $\zeta_{L}^{\prime} \neq \infty$ then on the horizon should be

$$
\begin{gathered}
\frac{p}{V} \zeta_{\perp}^{2}+\left.\frac{V}{p}\right|_{z=z_{h}}=0 \Rightarrow \zeta_{\perp}=i \frac{V\left(z_{h}\right)}{p} \\
\frac{1}{V} \zeta_{L}^{2}+\left.V\right|_{z=z_{h}}=0 \Rightarrow \zeta_{L}=i V\left(z_{h}\right)
\end{gathered}
$$


Since for $w=0$ the derivative $\zeta_{\perp}^{\prime}=0$ and we have

$$
\left.\zeta_{\perp}\right|_{z=0}=\left.\zeta_{\perp}\right|_{z=z_{h}}=\left.i \frac{V}{p}\right|_{z=z_{h}}
$$

$1 / z_{h}$ and it dependence on the temperature is read from (18) and approximately

$$
\frac{1}{z_{h}} \approx \frac{2 \pi v}{1+v} \frac{T}{1-\left(\frac{v}{v+1}\right)^{\frac{2+3 v}{v}} q^{2}\left(\frac{1}{2 \pi T}\right)^{\frac{2+4 v}{v}}}
$$

In what follows we use this approximation. Therefore, the dependence of the electric conductivity on the temperature and the chemical potential is given by

$$
\left|\zeta_{\perp}(T, v, q)\right| \approx\left(\frac{2 \pi v}{1+v}\right)^{3-2 / v} \frac{T^{3-2 / v}}{\left(1-\left(\frac{v+1 ! !}{v ! !}\right)^{\frac{2+3 v}{v}} q^{2}\left(\frac{1}{2 \pi T}\right)^{\frac{2+4 v}{v}}\right)^{3-2 / v}}
$$

Form this formula we see, that increasing anisotropy we increase the electric conductivity at hight temperatures, see Fig.13. We also see that there is a critical temperature $T_{0}=T_{0}(v, q)$ such that for $T<T_{0}$ the conductivity decreases as we increase $v$.
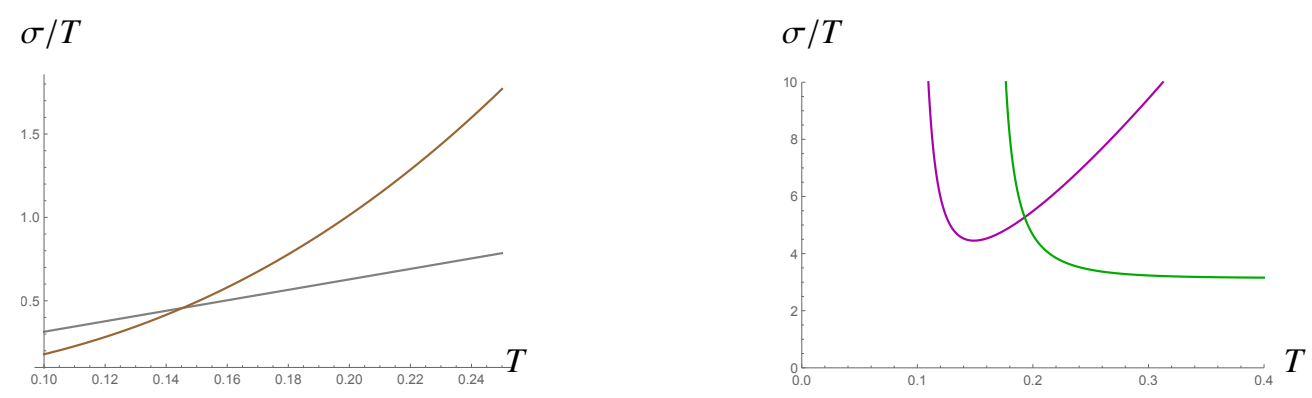

Figure 13. The electric conductivity for $v=1$ and $v=4$ and different values of $q$ : a) $q=0$, b) $q=0.2$

Here we have to note that equation (66) is valued only for $T>T_{a p p}$, see Fig.14. In Fig.14 and in Fig. 15 we plot the electric conductivity as function of $T$ for $v=1$ and $v=4$ for different values of $q$ and the constant $c$ specifying the factor $b$. We see that the confining factor $b(z)$ does not change the qualitative picture too much.

Note, that in the end of this sector few comments are in order. Almost all theoretical predictions underestimate the direct-photon spectra. Several attempts $[58,59]$, including the effects from strong magnetic fields [60], have been undertaken to fit theoretical predictions to LHC experimental data. As it is stressed in [56] direct photons calculations [53, 56, 61, 62] from holography have to be complemented by the medium evolution and the photons production from other phases. This also concerns to our consideration. Here we have just presented the preliminary estimation of the role of the chemical potential, the Lifshitz type anisotropy and the confining factor on photon emission rate. To give predictions for direct photons that can be observed at NICA one has to perform the study similar to [56]. We have seen that at hight temperature the anisotropy of the Lifshitz type increases the photon production and the chemical potential also increases it, meanwhile for temperature less then the critical one, they act to different direction. 


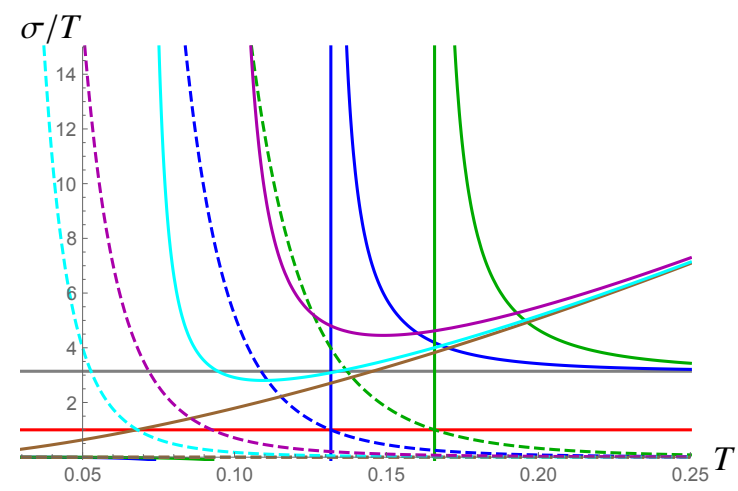

Figure 14. a) The electric conductivity for $v=1$ and $v=4$ and different values of $q$. The cases $v=1$ and $q=0,0.1,0.2$ are shown by gray, blue and green lines and the cases $v=4$ and $q=0,0.1,0.2$ are shown by brown, darker cyan and darker green. Dashed lines show validity of the approximation: only in the region where the dashed lines are below the red line we can use this approximation.

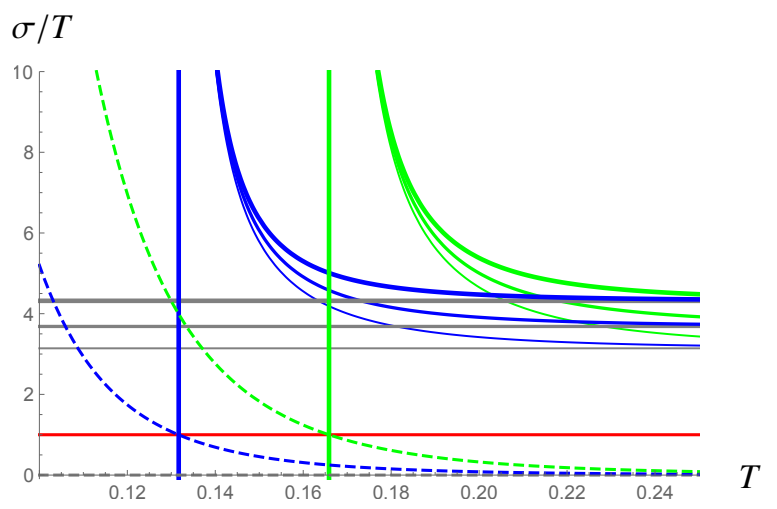

Figure 15. The electric conductivity for $v=1$, different values of $q$ and the constant $c$ specifying the factor $b$. The gray solid lines show the electric conductivity for $v=1$ and different values of $q=0,0.1,0.2$ (thin, middle and thick lines). The blue and green solid lines show the electric conductivity for $v=1$ and different values of $q=0,0.1,0.2$ (thin, middle and thick lines) and $c=1$ and $c=1.2$, respectively. The solid lines are the same as in a) and the dashed lines show validity of the approximation: only in the regions where the dashed lines are below the red line we can use our approximation.

\section{Conclusion}

As a conclusion let me summarize. Motivated by the fact that to fit the experimental form of dependence of total multiplicity on energy we have to deal with a special anisotropic holographic model, related with the Lifshitz-like background, we have estimated the holographic confinement/deconfinement phase transition in the $(\mu, T)$ plane in this anizotropic background. We have 


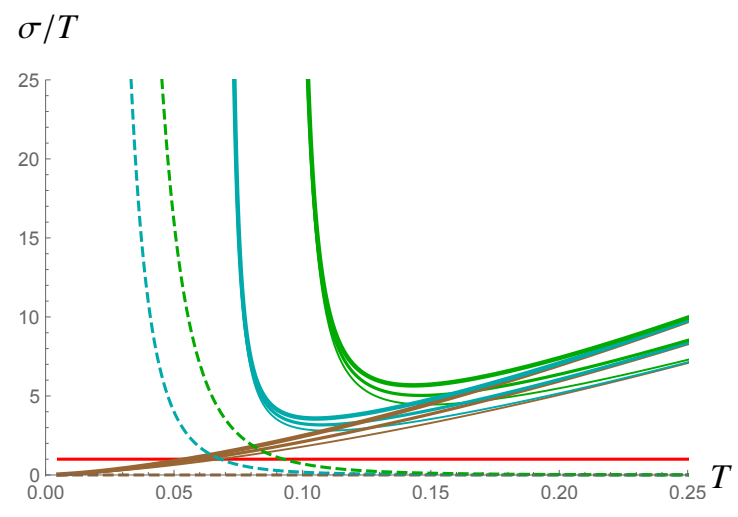

Figure 16. The electric conductivity for $v=4$, different values of $q$ and the constant $c$ specifying the factor $b$. The brown solid lines show the electric conductivity for $v=4$ and different values of $q=0,0.1,0.2$ (thin, middle and thick lines). The darker cyan and green solid lines show the electric conductivity for $v=4$ and different values of $q=0,0.1,0.2$ (thin, middle and thick lines) and $c=1$ and $c=1.2$, respectively. The dashed lines show validity of the approximation: only in the regions where the dashed lines are below the red line we can use our approximation.

found the dependence of the transition line on the orientation of the quark pair. This dependence leads to a non-sharp character of physical confinement/deconfinement phase in the $(\mu, T)$-plane. This calculation seems relevant in the context of the future project NICA and FAIR.

We are also going to estimate non-zero magnetic effects in the the Lifshitz-like background, in particular, by analogy with [63] one can estimate the crossover temperature in a magnetic field. As in isotropic case [64] it is interesting to find Debye screening mass near deconfinement in the anisotropic background.

\section{Acknowledgments}

I would like to thank the Organizers of ICNFP 2016 for the kind invitation and warm hospitality.

\section{References}

[1] J. Adams et al. (STAR Collab.) Nucl. Phys. A 757102 (2005)

[2] K. Aamodt et al. (ALICE Collab.) Phys. Rev. Lett. 105252301 (2010)

[3] E. Shuryak Nucl. Phys. A 75064 (2005)

[4] E.L. Feinberg, Phys. Usp. 168697 (1998)

[5] K. G. Wilson at al, Phys. Rev. D 49, 6720 (1994)

[6] O. Aharony et al. Phys. Rept. 323183 (2000)

[7] V. Kekelidze et al, Nucl.Phys.A,956 (2016) 846

[8] A. Bazavov, arXiv:1505.05543 [hep-lat].

[9] U. Gursoy, E. Kiritsis, L. Mazzanti and F. Nitti, JHEP 05, 033 (2009);

[10] O. DeWolfe, S. S. Gubser and C. Rosen, Phys. Rev. D 84, 126014 (2011) 
[11] J. Casalderrey-Solana, H. Liu, D. Mateos, K. Rajagopal, U. A. Wiedemann, Gauge/String Duality, Hot QCD and Heavy Ion Collisions, Cambridge University Press 2014

[12] G. Aad et al. [ATLAS Collaboration], Phys. Lett. B 710, 363 (2012)

[13] J. Adam et al. [ALICE Collaboration], Phys. Rev. Lett. 116, no. 22, 222302 (2016)

[14] I. Ya. Aref'eva, Phys. Usp. 57 (2014) 527.

[15] O. DeWolfe, S. S. Gubser, C. Rosen and D. Teaney, Prog. Part. Nucl. Phys. 75, 86 (2014).

[16] W. van der Schee, arXiv:1407.1849.

[17] S. S. Gubser, S. S. Pufu and A. Yarom, Phys. Rev. D 78 (2008) 066014; JHEP 0911 (2009) 050

[18] L. Alvarez-Gaume et al., JHEP 0902 (2009) 009

[19] S. Lin and E. Shuryak, Phys. Rev. D 79 (2009) 124015

[20] J. L. Albacete, Y. V. Kovchegov and A. Taliotis, JHEP 0905 (2009) 060

[21] I. Y. Aref'eva, A. A. Bagrov and E. A. Guseva, JHEP 0912, 009 (2009)

[22] I. Y. Aref'eva, A. A. Bagrov and L. V. Joukovskaya, JHEP 1003 , 002, (2010)

[23] Y. V. Kovchegov and S. Lin, JHEP 1003 (2010) 057; Y. V. Kovchegov, Prog. Theor. Phys. Suppl. 187 (2011) 96

[24] E. Kiritsis and A. Taliotis, JHEP 1204, 065 (2012)

[25] I. Ya. Aref'eva, E. O. Pozdeeva and T. O. Pozdeeva, Teor. Mat. Fiz. 180, 35 (2014).

[26] D. S. Ageev and I. Ya. Aref'eva, J. Exp. Theor. Phys. 120, no. 3, 436 (2015)

[27] L.D. Landau, Izvestya AN. Ser. phys. 1751 (1953)

[28] Fermi E Progr. Theoret. Phys. 5570 (1950)

[29] I.Ya. Pomeranchuk, DAN, SSSR 788841951

[30] I. Ya. Aref'eva and A. A. Golubtsova, JHEP 1504 (2015) (011).

[31] I. Y. Aref'eva, A. A. Golubtsova and E. Gourgoulhon, JHEP 1609, 142 (2016)

[32] O. Andreev and V. I. Zakharov, Phys. Rev. D 74, 025023 (2006)

[33] A. Chamblin, R. Emparan, C. V. Johnson and R. C. Myers, Phys. Rev. D 60064018 (1999)

[34] I. Ya. Arefeva, A. A. Bagrov and E. O. Pozdeeva, JHEP 1205, 117 (2012)

[35] D. Giataganas, PoS Corfu 2012, 122 (2013)

[36] M. Strickland, Pramana 84671 (2015).

[37] I. Aref'eva, EPJ Web Conf. 125, 01007 (2016).

[38] I. Ya. Aref'eva, Theor. Math. Phys., 184:3 (2015), 1239-1255

[39] D. S. Ageev, I. Y. Aref'eva, A. A. Golubtsova and E. Gourgoulhon, arXiv:1606.03995.

[40] D. Ageev, EPJ Web Conf. 125, 04007 (2016).

[41] J. M. Maldacena, Phys. Rev. Lett. 80, 4859 (1998);

[42] S. J. Rey, S. Theisen and J. T. Yee, Nucl. Phys. B 527, 171 (1998)

[43] A. Brandhuber, N. Itzhaki, J. Sonnenschein and S. Yankielowicz, Phys. Lett. B 434, 36 (1998)

[44] O. Andreev and V. I. Zakharov, JHEP 0704, 100 (2007);

[45] M. Mia, K. Dasgupta, C. Gale and S. Jeon, arXiv:1006.0055 [hep-th].

[46] P. Colangelo, F. Giannuzzi and S. Nicotri, Phys. Rev. D 83, 035015 (2011).

[47] R. G. Cai, S. He and D. Li, JHEP 1203, 033 (2012)

[48] Y. Yang and P. H. Yuan, JHEP 1512, 161 (2015)

[49] C. Ewerz, O. Kaczmarek and A. Samberg, arXiv:1605.07181 [hep-th].

[50] Z. Fang, S. He and D. Li,

[51] P. Braun-Munzinger and J. Stachel, Nucl. Phys. A 606, 320 (1996)

[52] A. Andronic, P. Braun-Munzinger, K. Redlich and J. Stachel, arXiv:1611.01347 [nucl-th]. 
[53] S. Caron-Huot, P. Kovtun, G. D. Moore, A. Starinets and L. G. Yaffe, JHEP 0612, 015 (2006)

[54] N. Iqbal and H. Liu, Phys. Rev. D 79, 025023 (2009)

[55] S. I. Finazzo and R. Rougemont, Phys. Rev. D 93, no. 3, 034017 (2016)

[56] I. Iatrakis, E. Kiritsis, C. Shen and D. L. Yang, arXiv:1609.07208 [hep-ph].

[57] I. Iatrakis, E. Kiritsis, C. Shen and D. L. Yang, arXiv:1611.04848 [hep-ph].

[58] L. McLerran and B. Schenke, Nucl. Phys. A 946, 158 (2016)

[59] J. F. Paquet, C. Shen, G. S. Denicol, M. Luzum, B. Schenke, S. Jeon and C. Gale, Phys. Rev. C 93, no. 4, 044906 (2016)

[60] B. Muller, S. Y. Wu and D. L. Yang, Phys. Rev. D 89, 026013 (2014)

[61] K. A. Mamo, JHEP 1308, 083 (2013)

[62] S. Y. Wu and D. L. Yang, JHEP 1308, 032 (2013)

[63] S. I. Finazzo and J. Noronha, Phys. Rev. D 90, 115028 (2014)

[64] R. Rougemont, R. Critelli and J. Noronha, Phys. Rev. D 93, 045013 (2016) 